\title{
Congruences on a Distributive Lattice
}

\author{
By H. A. Thurston
}

(Received 6th December, 1950. Read 12th January 1951.)

Among the many papers on the subject of lattices I have not seen any simple discussion of the congruences on a distributive lattice. It $i_{s}$ the purpose of this note to give such a discussion for lattices with a certain finiteness. Any distributive lattice is isomorphic with a ring of sets (G. Birkhoff, Lattice Theory, revised edition, 1948, p. 140, corollary to Theorem 6); I take the case where the sets are finite. All finite distributive lattices are covered by this case.

Let $\mathcal{L}$ be a lattice of subsets of $S$ and $K$ be a subset of $S$ (not necessarily an element of $\mathcal{L}$ ). Let the definition of the relation $\mathfrak{q}_{K}$ between elements of the lattice be that $X_{\mathfrak{q}_{K}} Y$ if and only if $X_{n} K=Y_{n} K . \quad q_{K}$ is clearly an equivalence (this would be true for any lattice). In fact, $\mathfrak{q}_{K}$ is a congruence. For if $X \mathfrak{q}_{K} Y$ and $Z \in L$, then $\left(X{ }_{n} Z\right)_{n} K=\left(X_{n} K\right)_{n} Z=\left(Y_{n} K\right)_{n} Z=\left(Y_{n} Z\right)_{n} K$ and $(X \cup Z)_{n} K=\left(X_{n} K\right) \cup\left(Z_{n} K\right)=\left(Y_{n} K\right) \cup\left(Z_{n} K\right)=(Y \cup Z)_{n} K$. Therefore $\left(X_{n} Z\right) \mathfrak{q}_{K}\left(Y_{n} Z\right)$ and $\left(X_{\cup} Z\right) \mathfrak{q}_{K}\left(Y_{\cup} Z\right)$.

The chief theorem is the converse of this: If $\mathcal{Q}$ is a lattice of finite subsets of $S$, and $q$ is a congruence on $\mathcal{L}$, then there is a subset $K$ of $S$ such that $\mathfrak{q}=\mathfrak{q}_{K}$.

The set $X \mathfrak{q}$ of all elements in the relation $q$ to $X$ is a sub-lattice. For if $Y_{\mathfrak{q}} X_{\mathfrak{q}} Z$, then $\left(Y_{\mathrm{n}} Z\right) \mathfrak{q}\left(X_{n} X\right)=X$ and $\left(Y_{\cup} Z\right) \mathfrak{q}\left(X_{\cup} X\right)=X$. Let $X_{g}$ and $X_{l}$ be the greatest and least elements of $X_{\mathfrak{q}}$.

Let $K$ be $\cup X-\cup\left(X_{q}-X_{l}\right)$. (Unions are over all $X$ of $\mathcal{L}$.) First we see that $q \subseteq \mathfrak{q}_{R}$. For $X_{g}-X_{l} \subseteq \cup X-K$. Therefore $\left(X_{g}-X_{l}\right)_{n} K \subseteq K-K=0$, and so $X_{g n} K=X_{l_{n}} K$. But $X_{g n} K \supseteq X_{n} K \supseteq X_{l n} K$, giving $X_{\cap} K=X_{l \cap} K$. If $X_{\mathfrak{q}}=Y \mathfrak{q}$ then $X_{l}=Y_{l}$. Therefore $Y_{n} K=Y_{l_{n}} K=X_{l_{n}} K=X_{n} K$, and so $X q_{K} Y$.

We have now to see that if $X_{\mathfrak{q}_{K}} Y$, then $X_{\mathfrak{q}} Y$. We take first the case $X_{2} Y$; the proof is by induction on the number of elements in $X-Y$. It is clearly true when $X-Y$ has no elements. Let $X-Y$ have $n$ elements $(n>0)$ and $a \in X-Y$ and $X_{n} K=Y_{n} K$. Then $a$ is not in $K$ and so $a \in U X-K=U\left(X_{v}-X_{l}\right)$. Therefore there are elements $P$ and $Q$ of $\mathcal{L}$ such that $P \mathfrak{q} Q$ and $a \in P-Q$. Then $U \mathfrak{q} V$ where $U=Y \cup P . \cup X$ and $V=Y_{\cup} Q \cdot{ }_{n} X$. 
Then

$$
Y \subseteq U_{n} V_{\subseteq} U \subseteq X
$$

Now $a$ is not in $U_{n} V$ and $a \in U$. The number of elements in $X-U$ and the number of elements in $\left(C_{n} V\right)-Y$ are therefore less than $n$. But, from (1), $K_{n} Y \subseteq K_{n} U_{n} V \subseteq K_{n} U \subseteq K_{n} X$. And $X_{n} K=Y_{n} K$. Therefore all these are equal. Therefore we have $K_{0} X=K_{0} U, U \subseteq X$, and the number of elements in $X-U$ is less than $n$. Therefore $U \mathfrak{q} X$. In the same way, $Y q U_{n} V$. But $U q U_{n} V$. Therefore $X q Y$.

Now let $(X, Y)$ be any element of $\mathfrak{q}_{K}$. Then $X_{n} K=X_{n} Y_{n} K$, and $X \supset X_{n} Y$, and so $X_{\mathfrak{q}} X_{n} Y$. In the same way, $Y_{\mathfrak{q}} Y_{n} X$, and so $X \mathfrak{q} Y$.

Note. A similar theorem for complemented modular lattices is given by Birkhoff, loc. cit., p. 119, Theorem 5.

Definition: If $\mathfrak{p}$ and $\mathfrak{r}$ are any two relations, then $\mathfrak{p r}$ is the relation for which $X \mathfrak{p r} Y$ if and only if there is a $Z$ for which $X \mathfrak{p} Z$ and $Z \mathrm{r} Y$.

We can now prove that

If $\mathfrak{p}$ and $\mathfrak{r}$ are any two congruences on a lattice of finite subsets, then $\mathrm{pr}=\mathfrak{r p}$.

Let $\mathfrak{p}$ be $\mathfrak{q}_{P}$ and $\mathfrak{r}$ be $\mathfrak{q}_{R}$. If $A \mathfrak{p r} B$, then, for some element $C$ of the lattice, $A q_{P} C q_{R} B$, and so $A_{n} P=C_{n} P$ and $B_{n} R=C_{n} R$.

Then $A_{n} P_{n} R=C_{n} P_{n} R=C_{n} R_{n} P=B_{n} R_{n} P$. Let $D$ be $\left(A_{n} R\right) \cup\left(B_{n} P\right)$. Then $D_{n} R=\left(A_{n} R\right)_{\cup}\left(B_{n} P_{n} R\right)=\left(A_{n} R\right) \cup\left(A_{n} P_{n} R\right)$ $=A_{n} R$. Therefore $A \times D$. In the same way, $D \mathfrak{p} B$. Therefore $A \mathfrak{r p} B$, and so $\mathfrak{p r} \subseteq \mathfrak{r p}$. Similarly, $\mathfrak{i p} \subseteq \mathfrak{p r}$.

Note. This theorem was proved for relatively complemented lattices by R. P. Dilworth, Annals of Mathematics, 50(1950), 348.

\section{The University,} BrISTOL. 